Journal homepage: http://dergipark.ulakbim.gov.tr/jotcsa

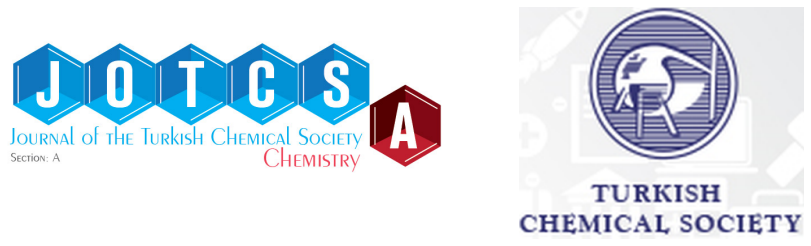

e-ISSN: $2149-0120$

\title{
CHEMICAL REACTIVITY OF ETHYL 4-(1-ETHYL-1,2-DIHYDRO-4- HYDROXY-2-OXOQUINOLIN-3-YL)-2,4-DIOXOBUTANOATE TOWARDS SOME NUCLEOPHILIC REAGENTS
}

\author{
Magdy A. Ibrahim ${ }^{1}$, Hany M. Hassanin ${ }^{1 *}$, and Shimaa Badran ${ }^{1}$ \\ ${ }^{1}$ Department of Chemistry, Faculty of Education, Ain Shams University, Roxy, Heliopolis \\ 11757, Cairo, Egypt.
}

\begin{abstract}
A novel series of heterocyclic systems linked with quinolin-2-one was efficiently synthesized from reaction of ethyl 4-(1-ethyl-1,2-dihydro-4-hydroxy-2oxoquinolin-3-yl)-2,4-dioxobutanoate (1) with a variety of nitrogen and/or carbon nucleophiles. A variety of heterocyclic systems such as pyrazoles, pyrimidines, pyrazines, oxazines and triazines containing quinoline moiety were synthesized. Structures of the new synthesized products were deduced on basis of their analytical and spectral data.
\end{abstract}

Keywords: Quinolin-2-one, diketoester, active methylene, nucleophilic reaction, heterocyclization.

Submitted: December 12, 2015. Revised: January 11, 2016. Accepted: February 7, 2016.

Cite this: Ibrahim M, Hassanin H, Badran S. CHEMICAL REACTIVITY OF ETHYL 4-(1-ETHYL-1,2DIHYDRO-4-HYDROXY-2-OXOQUINOLIN-3-YL)-2,4-DIOXOBUTANOATE TOWARDS SOME NUCLEOPHILIC REAGENTS. Journal of the Turkish Chemical Society, Section A: Chemistry. 2016;3(2):75-88.

DOI: $10.18596 /$ jotcsa.11975.

Correspondence to: Hany M. Hassanin. E-mail: hanyhassnin@yahoo.com. 


\section{INTRODUCTION}

4-Hydroxyquinolin-2(1H)-ones represent one of the most important classes of heterocycles possessing a wide spectrum of biological activities [1-4]. Quinoline derivatives have been used as bactericidal [5], anti-inflammatory [6], antimalarial [7], antitumor [8], antioxidant [9], antileishmanial [10] and antiplatelet activities [11] such as DNA binding capabilities and DNA-intercalating carrier [12]. Also, a broad number of important pharmacological activities have been associated with 3-substituted 4-hydroxyquinolin-2(1H)-ones [13, 14]. The present work aimed to synthesize ethyl 4-(1-ethyl-4-hydroxy-2-oxo-1,2-dihydroquinolin-3-yl)-2,4dioxobutyrate (1) [15] as a starting material and study its chemical reactivity towards a variety of nucleophilic reagents, in a hope to obtain a novel series 4-hydroxyquinolin-2(1H)ones bearing variable heterocyclic systems of expected biological activity.

\section{EXPERIMENTAL SECTION}

General. Melting points were determined on a digital Stuart SMP3 apparatus. Fouriertransform infrared spectra were taken on FT-IR Nicolet IS10 spectrophotometer $\left(\mathrm{cm}^{-1}\right)$, using $\mathrm{KBr}$ disks. ${ }^{1} \mathrm{H}$ NMR (300 MHz) spectra were measured on Mercury-300BB, using DMSO- $\mathrm{d}_{6}$ as a solvent and tetramethylsilane as an internal standard. Mass spectra were measured using GC-2010 Shimadzu Gas chromatography instrument mass spectrometer (70 eV) GC-MS qp 1000 ex Shimadzu instrument $(70 \mathrm{eV})$. Elemental microanalyses were performed on a Perkin-Elmer CHN-2400 analyzer at the Chemical War Department, Ministry of Defense, Egypt. Ethyl 4-(1-ethyl-4-hydroxy-2-oxo-1,2-dihydroquinolin-3-yl)-2,4-dioxobutyrate (3) [15] was prepared according to the literature method.

\section{Ethyl 1-(2-cyanoacetyl)-5-(1-ethyl-1,2-dihydro-4-hydroxy-2-oxoquinolin-3-yl)-1H- pyrazole-3-carboxylate (3).}

A mixture of diketoester $1(0.50 \mathrm{~g}, 1.51 \mathrm{mmol})$ and cyanoacetohydrazide $(0.15 \mathrm{~g}, 1.51$ $\mathrm{mmol})$, in absolute ethanol $(20 \mathrm{~mL})$, containing few drops of acetic acid, was stirred at room temperature for $2 \mathrm{~h}$. The beige precipitate so formed was filtered and crystallized from EtOH to give compound 3 as beige crystals, yield $(0.30 \mathrm{~g}, 54 \%)$, m.p. $>300{ }^{\circ} \mathrm{C}$. FT-IR $\left(\mathrm{KBr}, \mathrm{cm}^{-}\right.$ $\left.{ }^{1}\right)$ : $3446(\mathrm{OH}), 2987,2934\left(\mathrm{CH}_{\text {aliph. }}\right), 2263(\mathrm{C} \equiv \mathrm{N}), 1756\left(\mathrm{C}=\mathrm{O}_{\text {ester }}\right), 1695\left(\mathrm{C}=\mathrm{O}_{\text {ketone }}\right), 1638$ $\left(\mathrm{C}=\mathrm{O}_{\text {quinolone }}\right), 1572(\mathrm{C}=\mathrm{N})$ and $1504(\mathrm{C}=\mathrm{C}) .{ }^{1} \mathrm{H} \mathrm{NMR}\left(\delta, 300 \mathrm{MHz}, \mathrm{DMSO}-\mathrm{d}_{6}\right): 1.16-1.32$ (m, $\left.6 \mathrm{H}, 2 \mathrm{CH}_{2} \mathrm{CH}_{3}\right), 3.90\left(\mathrm{~s}, 2 \mathrm{H}, \mathrm{CH}_{2}\right), 4.19\left(\mathrm{q}, 2 \mathrm{H}, \mathrm{NCH}_{2} \mathrm{CH}_{3}\right), 4.48\left(\mathrm{q}, 2 \mathrm{H}, \mathrm{OCH}_{2} \mathrm{CH}_{3}\right), 7.20(\mathrm{~s}$, $\left.1 \mathrm{H}, \mathrm{H}-4_{\text {pyrazole }}\right), 7.32\left(\mathrm{t}, 1 \mathrm{H}, \mathrm{H}-6_{\text {quinolone }}\right), 7.59\left(\mathrm{~d}, 1 \mathrm{H}, \mathrm{H}-8_{\text {quinolone }}\right), 7.72\left(\mathrm{t}, 1 \mathrm{H}, \mathrm{H}-7_{\text {quinolone }}\right)$, $8.06\left(\mathrm{~d}, 1 \mathrm{H}, \mathrm{H}-5_{\text {quinolone }}\right), 11.90$ (bs, $1 \mathrm{H}, \mathrm{OH}$ exchangeable with $\left.\mathrm{D}_{2} \mathrm{O}\right)$. Mass spectrum, $\mathrm{m} / \mathrm{z}\left(\mathrm{I}_{\mathrm{r}}\right.$ \%): $394\left(\mathrm{M}^{+}, 14\right), 393(60), 363$ (24), 368 (11), 327 (17), 268 (47), 230 (10), 212 (18), 197 (16), 188 (8), 172 (8), 161 (8), 146 (19), 132 (29), 119 (21), 102 (66), 91 (25), 77 (79), 64 (96) and 55 (100). Anal. Calcd. for $\mathrm{C}_{19} \mathrm{H}_{19} \mathrm{~N}_{3} \mathrm{O}_{5}$ (394.38); C, 60.91; H, 4.60; N, $14.21 \%$. Found: $\mathrm{C}, 60.80 ; \mathrm{H}, 4.55 ; \mathrm{N}, 14.08 \%$. 


\section{Ethyl 5-(1-ethyl-1,2-dihydro-4-hydroxy-2-oxoquinolin-3-yl)-1-[(pyridine-3-yl) carbonyl]-1H-pyrazole-3-carboxylate (4).}

A mixture of diketoester $1(0.50 \mathrm{~g}, 1.51 \mathrm{mmol})$ and nicotinic acid hydrazide $(0.21 \mathrm{~g}, 1.51$ $\mathrm{mmol})$, in absolute ethanol $(20 \mathrm{~mL})$, containing few drops of acetic acid, was heated under reflux for $2 \mathrm{~h}$. The brown crystals deposited after cooling were filtered and recrystallized from ethanol to give compound 4 as pale brown crystals, yield $(0.24 \mathrm{~g}, 37 \%)$, m.p. > 300 ${ }^{\circ} \mathrm{C}$; FT-IR $(\mathrm{KBr}) \mathrm{v}_{\max } 3446(\mathrm{OH}), 2981,2931\left(\mathrm{CH}_{\text {aliph. }}\right), 1706\left(\mathrm{C}=\mathrm{O}_{\text {ester }}\right), 1683\left(\mathrm{C}=\mathrm{O}_{\text {ketone }}\right)$, $1632\left(\mathrm{C}=\mathrm{O}_{\text {quinolone }}\right), 1617(\mathrm{C}=\mathrm{N})$ and $1550 \mathrm{~cm}^{-1}(\mathrm{C}=\mathrm{C})$; ${ }^{1} \mathrm{H}$ NMR (DMSO-d $\left.{ }_{6}, 300 \mathrm{MHz}\right) \delta 1.18-$ $1.30\left(\mathrm{~m}, 6 \mathrm{H}, 2 \mathrm{CH}_{2} \mathrm{CH}_{3}\right), 4.10-4.25\left(\mathrm{~m}, 4 \mathrm{H}, 2 \mathrm{CH}_{2} \mathrm{CH}_{3}\right), 7.10\left(\mathrm{~s}, 1 \mathrm{H}, \mathrm{H}-4_{\text {pyrazole }}\right), 7.40-7.60$ $\left(\mathrm{m}, 3 \mathrm{H}, \mathrm{H}-6_{\text {quinolone, }} \mathrm{H}-8_{\text {quinolone }}\right.$ and $\left.\mathrm{H}-\mathrm{7}_{\text {quinolone }}\right), 8.10$ (d, $\left.1 \mathrm{H}, \mathrm{H}-5_{\text {quinolone }}\right), 8.30(\mathrm{t}, 1 \mathrm{H}, \mathrm{H}-$ $\left.5_{\text {pyridine }}\right), 8.60-8.80\left(\mathrm{~m}, 2 \mathrm{H}, \mathrm{H}-4_{\text {pyridine }}\right.$ and $\left.\mathrm{H}-6_{\text {pyridine }}\right), 8.90\left(\mathrm{~s}, 1 \mathrm{H}, \mathrm{H}-2_{\text {pyridine }}\right), 10.00$ (bs, $1 \mathrm{H}$, $\mathrm{OH}$ exchangeable with $\mathrm{D}_{2} \mathrm{O}$ ); Mass spectrum, $\mathrm{m} / \mathrm{z}$ (rel. int. \%): $432\left[\mathrm{M}^{+} \cdot\right](6), 347$ (3), 327 (6), 281 (5), 269 (4), 224 (4), 182 (9), 132 (6), 120 (5), 106 (37) and 64 (100). Anal. Calcd for $\mathrm{C}_{23} \mathrm{H}_{20} \mathrm{~N}_{4} \mathrm{O}_{5}$ (432.44), C, 63.90\%; $\mathrm{H}, 4.50 \%$; N, 12.88\%; Found, C, 63.88\%, H, 4.66\%, $\mathrm{N}, 12.96 \%$.

\section{Ethyl 1-(7-chloroquinolin-4-yl)-5-(1-ethyl-1,2-dihydro-4-hydroxy-2-oxoquinolin-3- yl)-1H-pyrazole-3-carboxylate (5).}

A mixture of diketoester $1(0.50 \mathrm{~g}, 1.51 \mathrm{mmol})$ and 7-chloro-4-hydrazinoquinoline (2) (0.29 $\mathrm{g}, 1.51 \mathrm{mmol})$, in absolute ethanol $(20 \mathrm{~mL})$, containing few drops of acetic acid, was heated under reflux for $4 \mathrm{~h}$. The reaction mixture was left to cool at room temperature. The solid so formed was filtered and crystallized from DMF to give compound $\mathbf{5}$ as red-brown crystals, yield $(0.35 \mathrm{~g}, 47 \%)$, m.p. $>300{ }^{\circ} \mathrm{C}$; FT-IR $(\mathrm{KBr}) \mathrm{v}_{\max } 3406(\mathrm{OH}), 3076\left(\mathrm{CH}_{\text {arom. }}\right), 2931$ $\left(\mathrm{CH}_{\text {aliph. }}\right), 1707\left(\mathrm{C}=\mathrm{O}_{\text {ester }}\right), 1628\left(\mathrm{C}=\mathrm{O}_{\text {quinolone }}\right), 1608(\mathrm{C}=\mathrm{N})$ and $1586 \mathrm{~cm}^{-1}(\mathrm{C}=\mathrm{C}) ;{ }^{1} \mathrm{H}$ NMR (DMSO- $\left.\mathrm{d}_{6}, 300 \mathrm{MHz}\right) \delta 1.19-1.22\left(\mathrm{~m}, 6 \mathrm{H}, 2 \mathrm{CH}_{2} \mathrm{CH}_{3}\right), 4.10-4.30\left(\mathrm{~m}, 4 \mathrm{H}, 2 \mathrm{CH}_{2} \mathrm{CH}_{3}\right), 6.40(\mathrm{~s}$, $\left.1 \mathrm{H}, \mathrm{H}-4_{\text {pyrazole }}\right), 7.12\left(\mathrm{t}, 1 \mathrm{H}, \mathrm{H}-6_{\text {quinolone }}\right), 7.32-7.46\left(\mathrm{~m}, 2 \mathrm{H}, \mathrm{H}-8_{\text {quinolone }}\right.$ and $\left.\mathrm{H}-6_{\text {quinoline }}\right), 7.46$ ( $\left.\mathrm{s}, 1 \mathrm{H}, \mathrm{H}-8_{\text {quinoline }}\right), 7.6\left(\mathrm{t}, 1 \mathrm{H}, \mathrm{H}-7_{\text {quinolone }}\right), 7.60-8.00\left(\mathrm{~m}, 2 \mathrm{H}, \mathrm{H}-5_{\text {quinolone }}\right.$ and $\left.\mathrm{H}-5_{\text {quinoline }}\right), 8.15$ (d, $\left.1 \mathrm{H}, \mathrm{H}-2_{\text {quinoline }}\right), 8.32\left(\mathrm{~d}, 1 \mathrm{H}, \mathrm{H}-3_{\text {quinoline }}\right)$; Mass spectrum, $\mathrm{m} / \mathrm{z}$ (rel. int. \%): 489 [M $\mathrm{M}^{+}$] (67), 464 (91), 432 (77), 417 (97), 352 (80), 319 (83), 272 (65), 229 (95), 206 (97), 178 (100) and 77 (76). Anal. Calcd for $\mathrm{C}_{26} \mathrm{H}_{21} \mathrm{CIN}_{4} \mathrm{O}_{4}$ (488.93), C 63.70\%; H 4.40\%; N 11.36\%; found, C $63.87 \%$; $\mathrm{H} 4.33 \%$; N $11.46 \%$.

\section{Ethyl 5-(1-ethyl-1,2-dihydro-4-hydroxy-2-oxoquinolin-3-yl)[1,2,4]triazolo[4,3-a] pyrimidine-7-carboxylate (7).}

A mixture of diketoester $1(0.50 \mathrm{~g}, 1.51 \mathrm{mmol})$ and 3-amino[1,2,4]triazole (6) $(0.13 \mathrm{~g}, 1.51$ $\mathrm{mmol})$, in absolute ethanol $(20 \mathrm{~mL}$ ) containing few drops of acetic acid, was heated under reflux for $4 \mathrm{~h}$. The yellow crystals deposited after cooling were filtered and crystallized from EtOH to give compound 7 as golden yellow crystals, yield $\left(0.38\right.$ g, 67\%), m.p. $200-201{ }^{\circ} \mathrm{C}$; FT-IR ( KBr) $v_{\max } 3447(\mathrm{OH}), 3103\left(\mathrm{CH}_{\text {arom. }}\right), 2970,2925,2854\left(\mathrm{CH}_{\text {aliph. }}\right), 1751\left(\mathrm{C}=\mathrm{O}_{\text {ester }}\right)$, $1635\left(\mathrm{C}=\mathrm{O}_{\text {quinolone }}\right), 1609(\mathrm{C}=\mathrm{N})$ and $1570 \mathrm{~cm}^{-1}(\mathrm{C}=\mathrm{C}) .{ }^{1} \mathrm{H} \mathrm{NMR}\left(\mathrm{DMSO}-\mathrm{d}_{6}, 300 \mathrm{MHz}\right) \delta 1.21$ $\left(\mathrm{t}, 3 \mathrm{H}, J=6.9 \mathrm{~Hz}, \mathrm{~N}-\mathrm{CH}_{2} \mathrm{CH}_{3}\right), 1.38\left(\mathrm{t}, 3 \mathrm{H}, J=6.7 \mathrm{~Hz}, \mathrm{O}-\mathrm{CH}_{2} \mathrm{CH}_{3}\right), 4.24(\mathrm{q}, 2 \mathrm{H}, J=6.9 \mathrm{~Hz}, \mathrm{~N}-$ $\left.\mathrm{CH}_{2} \mathrm{CH}_{3}\right), 4.50\left(\mathrm{q}, 2 \mathrm{H}, \mathrm{J}=6.7 \mathrm{~Hz}, \mathrm{O}-\mathrm{CH}_{2} \mathrm{CH}_{3}\right), 7.20\left(\mathrm{t}, 1 \mathrm{H}, \mathrm{H}-6_{\text {quinolone }}\right), 7.51(\mathrm{~d}, 1 \mathrm{H}, \mathrm{H}-$ $\left.8_{\text {quinolone }}\right), 7.60\left(\mathrm{t}, 1 \mathrm{H}, \mathrm{H}-7_{\text {quinolone }}\right), 8.03\left(\mathrm{~d}, 1 \mathrm{H}, \mathrm{J}=6.7 \mathrm{~Hz}, \mathrm{H}-5_{\text {quinolone }}\right), 8.73(\mathrm{~s}, 1 \mathrm{H}, \mathrm{H}-$ $\left.5_{\text {pyrimidine }}\right), 9.40\left(\mathrm{~s}, 1 \mathrm{H}, \mathrm{H}-3_{\text {triazole }}\right), 17$ (bs, $1 \mathrm{H}, \mathrm{OH}$ exchangeable with $\left.\mathrm{D}_{2} \mathrm{O}\right)$. Mass spectrum, m/z (rel. int. \%): 379 [M+.] (95), 360 (61), 331 (71), 279 (67), 267 (67), 223 (84), 203 (61), 162 (69), 132 (100), 89 (47) and 77 (84). Anal. Calcd for $\mathrm{C}_{19} \mathrm{H}_{17} \mathrm{~N}_{5} \mathrm{O}_{4}$ (379.38), C, $60.20 \% ; H, 4.65 \%$; N, $18.52 \%$; found, C 60.15\%; H 4.52\%; N 18.46\%. 


\section{Ethyl 4-(1-ethyl-1,2-dihydro-4-hydroxy-2-oxoquinolin-3-yl)pyrimido[1,2-a] benzimidazole-2-carboxylate (9).}

A mixture of diketoester $1(0.50 \mathrm{~g}, 1.51 \mathrm{mmol})$ and 2-aminobenzimidazole (8) $(0.20 \mathrm{~g}, 1.51$ $\mathrm{mmol})$, in absolute ethanol ( $20 \mathrm{~mL})$, containing few drops of acetic acid, was heated under reflux for $2 \mathrm{~h}$. The orange-red crystals obtained during heating was filtered and crystallized from DMF to give compound 9 as orange-red, yield $(0.50 \mathrm{~g}, 77 \%)$, m.p. $>300{ }^{\circ} \mathrm{C}$; FT-IR $(\mathrm{KBr}) \mathrm{v}_{\max } 3359(\mathrm{OH}), 3093\left(\mathrm{CH}_{\text {arom. }}\right), 2970,2925\left(\mathrm{CH}_{\text {aliph. }}\right), 1707\left(\mathrm{C}=\mathrm{O}_{\text {ester }}\right), 1645$ $\left(\mathrm{C}=\mathrm{O}_{\text {quinolone }}\right), 1612(\mathrm{C}=\mathrm{N})$ and $1588 \mathrm{~cm}^{-1}(\mathrm{C}=\mathrm{C}) ;{ }^{1} \mathrm{H}$ NMR (DMSO-d $\left.{ }_{6}, 300 \mathrm{MHz}\right) \delta 1.19-1.24$ $\left(\mathrm{m}, 6 \mathrm{H}, 2 \mathrm{CH}_{2} \mathrm{CH}_{3}\right), 4.20-4.30\left(\mathrm{~m}, 4 \mathrm{H}, 2 \mathrm{CH}_{2} \mathrm{CH}_{3}\right), 6.90(\mathrm{~s}, 1 \mathrm{H}, \mathrm{H}-3$ pyrimidine $), 7.02-7.04(\mathrm{~m}, 2 \mathrm{H}$, $\operatorname{Ar}-\mathrm{H}), 7.22-7.24(\mathrm{~m}, 2 \mathrm{H}, \mathrm{Ar}-\mathrm{H}), 7.41\left(\mathrm{t}, 1 \mathrm{H}, J=7.5 \mathrm{~Hz}, \mathrm{H}-6_{\text {quinolone }}\right), 7.64(\mathrm{~d}, 1 \mathrm{H}, J=8.7 \mathrm{~Hz}$, $\left.\mathrm{H}-8_{\text {quinolone }}\right), 7.79(\mathrm{t}, 1 \mathrm{H}, J=7.5 \mathrm{~Hz}, \mathrm{H}-7$ quinolone $), 8.26\left(\mathrm{~d}, 1 \mathrm{H}, J=7.8 \mathrm{~Hz}, \mathrm{H}-5_{\text {quinolone }}\right)$; Mass spectrum, m/z (rel. int. \%): 428 [M+.] (83), 386 (100), 346 (65), 296 (77), 233 (90), 177 (87), 134 (90), 109 (48), 92 (76) and 70 (75). Anal. Calcd for $\mathrm{C}_{24} \mathrm{H}_{20} \mathrm{~N}_{4} \mathrm{O}_{4}$ (428.45), C, $67.17 \% ; \mathrm{H}, 4.82 \% ; \mathrm{N}, 13.10 \%$; found, $\mathrm{C}, 67.28 \% ; \mathrm{H}, 4.71 \% ; \mathrm{N}, 13.08 \%$.

\section{1-Ethyl-3-[2-(3,4,5,6-tetrahydro-3-oxopyrazin-3-yl)acetyl]-4-hydroxyquinolin- 2(1H)-one (10).}

A mixture of diketoester $1(0.50 \mathrm{~g}, 1.51 \mathrm{mmol})$ and ethylene diamine $(0.09 \mathrm{~mL})$, in absolute ethanol $(20 \mathrm{~mL})$ containing few drops of acetic acid, was heated under reflux for $2 \mathrm{~h}$. The solid obtained after cooling was filtered and crystallized from EtOH to give compound $\mathbf{1 0}$ as brown crystals, yield $(0.21 \mathrm{~g}, 41 \%)$, m.p. > $300{ }^{\circ} \mathrm{C}$; FT-IR (KBr) $v_{\max } 3425(\mathrm{OH}), 3340$ $(\mathrm{NH}), 3067\left(\mathrm{CH}_{\text {arom. }}\right), 2975,2936\left(\mathrm{CH}_{\text {aliph. }}\right), 1675\left(\mathrm{C}=\mathrm{O}_{\text {amide }}\right), 1632\left(\mathrm{C}=\mathrm{O}_{\text {ketone }}\right.$ and $\left.\mathrm{C}=\mathrm{O}_{\text {quinolone }}\right)$ and $1559 \mathrm{~cm}^{-1}(\mathrm{C}=\mathrm{N}$ and $\mathrm{C}=\mathrm{C}) ;{ }^{1} \mathrm{H} \mathrm{NMR}\left(\mathrm{DMSO}-\mathrm{d}_{6}, 300 \mathrm{MHz}\right) \delta 1.16(\mathrm{t}, 3 \mathrm{H}$, $\left.\mathrm{CH}_{2} \mathrm{CH}_{3}\right), 2.70\left(\mathrm{~s}, 2 \mathrm{H}, \mathrm{CH}_{2}\right), 3.21-3.30\left(\mathrm{~m}, 4 \mathrm{H}, \mathrm{N}-\mathrm{CH}_{2}-\mathrm{CH}_{2}-\mathrm{N}\right), 4.35\left(\mathrm{q}, 2 \mathrm{H}, \mathrm{CH}_{2} \mathrm{CH}_{3}\right), 7.16(\mathrm{t}$, $\left.1 \mathrm{H}, J=6.0 \mathrm{~Hz}, \mathrm{H}-6_{\text {quinolone }}\right), 7.36\left(\mathrm{~d}, 1 \mathrm{H}, J=7.2 \mathrm{~Hz}, \mathrm{H}-8_{\text {quinolone }}\right), 7.60\left(\mathrm{t}, 1 \mathrm{H}, \mathrm{H}-7_{\text {quinolone }}\right), 8.07$ (d, $1 \mathrm{H}, J=7.8 \mathrm{~Hz}, \mathrm{H}-5_{\text {quinolone }}$ ), 8.70 (bs, $1 \mathrm{H}, \mathrm{NH}$ exchangeable with $\mathrm{D}_{2} \mathrm{O}$ ). Mass spectrum, $\mathrm{m} / \mathrm{z}\left(\mathrm{I}_{\mathrm{r}} \%\right): 327\left(\mathrm{M}^{+}, 1\right), 246(63), 233(24), 216(100), 189(45), 172(4), 146(14), 132$ (28), 119 (13), 91 (9), 77 (66) and 64 (50). Anal. Calcd for $\mathrm{C}_{17} \mathrm{H}_{17} \mathrm{~N}_{3} \mathrm{O}_{4}$ (327.34), C, $62.31 \% ; \mathrm{H}, 5.31 \% ; \mathrm{N}, 12.89 \%$; found, $\mathrm{C}, 62.38 ; \mathrm{H}, 5.23 ; \mathrm{N}, 12.84 \%$.

\section{1-Ethyl-3-[2-(1,2-dihydro-2-oxoquinoxalin-3-yl)acetyl]-4-hydroxyquinolin-2(1H)- one (11).}

A mixture of diketoester $1(0.50 \mathrm{~g}, 1.51 \mathrm{mmol})$ and o-phenylene diamine $(0.16 \mathrm{~g}, 1.51$ $\mathrm{mmol})$ in absolute ethanol $(20 \mathrm{~mL})$, containing few drops of acetic acid, was heated under reflux for $2 \mathrm{~h}$. The orange crystals obtained during heating were filtered and crystallized from DMF to give compound $\mathbf{1 1}$ as pale orange crystals, yield $(0.33 \mathrm{~g}, 62 \%)$, m.p. > $300{ }^{\circ} \mathrm{C}$; FTIR $(\mathrm{KBr}) \mathrm{v}_{\max } 3447(\mathrm{OH}), 3121(\mathrm{NH}), 3050\left(\mathrm{CH}_{\text {arom. }}\right), 2931\left(\mathrm{CH}_{\text {aliph. }}\right), 1685\left(\mathrm{C}=\mathrm{O}_{\text {amide }}\right), 1647$ $\left(\mathrm{C}=\mathrm{O}_{\text {ketone }}\right.$ and $\left.\mathrm{C}=\mathrm{O}_{\text {quinolone }}\right), 1601(\mathrm{C}=\mathrm{N})$ and $1559 \mathrm{~cm}^{-1}(\mathrm{C}=\mathrm{C}) \cdot{ }^{1} \mathrm{H}$ NMR (DMSO- $\left.\mathrm{d}_{6}, 300 \mathrm{MHz}\right)$ $\delta 1.18\left(\mathrm{t}, 3 \mathrm{H}, \mathrm{CH}_{2} \mathrm{CH}_{3}\right), 2.60\left(\mathrm{~s}, 2 \mathrm{H}, \mathrm{CH}_{2}\right), 4.24\left(\mathrm{q}, 2 \mathrm{H}, \mathrm{CH}_{2} \mathrm{CH}_{3}\right), 7.01-8.10(\mathrm{~m}, 8 \mathrm{H}, \mathrm{Ar}-\mathrm{H})$, 12.10 (bs, $1 \mathrm{H}, \mathrm{NH}$ exchangeable with $\mathrm{D}_{2} \mathrm{O}$ ), 12.80 (bs, $1 \mathrm{H}, \mathrm{OH}$ exchangeable with $\mathrm{D}_{2} \mathrm{O}$ ); Mass spectrum, m/z (rel. int. \%): 375 [M+.] (10), 347 (4), 329 (3), 261 (3), 216 (6), 189 (9), 160 (15), 132 (22), $119(6), 103$ (6) and 64 (100). Anal. Calcd for $\mathrm{C}_{21} \mathrm{H}_{17} \mathrm{~N}_{3} \mathrm{O}_{4}$ (375.39), C, 67.28\%; H, 4.45\%; N, $11.32 \%$; found, C, 67.19\%; H, 4.56\%; N, $11.19 \%$. 


\section{1-Ethyl-3-[2-(2-oxo-2H-benzo[b][1,4]-oxazin-3-yl)acetyl]-4-hydroxyquinolin- 2(1H)-one (12).}

A mixture of diketoester $1(0.50 \mathrm{~g}, 1.51 \mathrm{mmol})$ and 2-aminophenol $(0.16 \mathrm{~g}, 1.51 \mathrm{mmol})$, in absolute ethanol $(20 \mathrm{~mL})$, containing few drops of acetic acid, was heated under reflux for 2 $\mathrm{h}$. The solid obtained during heating was filtered and crystallized from DMF to give compound 12 as orange crystals, yield $\left(0.2\right.$ g, 37\%), m.p. 260-261 ${ }^{\circ} \mathrm{C}$; FT-IR $(\mathrm{KBr}) \mathrm{v}_{\max }$ $3140(\mathrm{OH}), 3050\left(\mathrm{CH}_{\text {arom. }}\right), 2928\left(\mathrm{CH}_{\text {aliph. }}\right), 1752\left(\mathrm{C}=\mathrm{O}_{\text {a-pyrone }}\right), 1641\left(\mathrm{C}=\mathrm{O}_{\text {ketone }}\right.$ and $\left.\mathrm{C}=\mathrm{O}_{\text {quinolone }}\right), 1604(\mathrm{C}=\mathrm{N})$ and $1560 \mathrm{~cm}^{-1}(\mathrm{C}=\mathrm{C}) ;{ }^{1} \mathrm{H} \mathrm{NMR}\left(\mathrm{DMSO}-\mathrm{d}_{6}, 300 \mathrm{MHz}\right) \delta 1.22(\mathrm{t}, 3 \mathrm{H}$, $\left.J=6.6 \mathrm{~Hz}, \mathrm{CH}_{2} \mathrm{CH}_{3}\right), 2.60\left(\mathrm{~s}, 2 \mathrm{H}, \mathrm{CH}_{2}\right), 4.26\left(\mathrm{q}, 2 \mathrm{H}, J=6.6 \mathrm{~Hz}, \mathrm{CH}_{2} \mathrm{CH}_{3}\right), 7.15-7.26(\mathrm{~m}, 3 \mathrm{H}$, $\operatorname{Ar}-\mathrm{H}), 7.50(\mathrm{~d}, 1 \mathrm{H}, J=8.1 \mathrm{~Hz}, \mathrm{Ar}-\mathrm{H}), 7.69(\mathrm{~d}, 1 \mathrm{H}, J=6.9 \mathrm{~Hz}, \mathrm{Ar}-\mathrm{H}), 7.76(\mathrm{t}, 1 \mathrm{H}, J=7.8 \mathrm{~Hz}$, Ar- $\mathrm{H}), 8.12-8.20(\mathrm{~m}, 2 \mathrm{H}, \mathrm{Ar}-\mathrm{H}), 12.14$ (bs, $1 \mathrm{H}, \mathrm{OH}$ exchangeable with $\left.\mathrm{D}_{2} \mathrm{O}\right)$; Mass spectrum, m/z (rel. int. \%): 376 [M+.] (17), 348 (21), 313 (21), 298 (23), 223 (18), 188 (21), 154 (22), $133(100), 120$ (64), 104 (89) and 77 (33). Anal. Calcd for $\mathrm{C}_{21} \mathrm{H}_{16} \mathrm{~N}_{2} \mathrm{O}_{5}$ (376.37), C, 67.10\%; H, 4.38\%; N, 7.54\%; found, C, 67.02\%; H, 4.28\%; N, 7.44\%.

\section{5-Ethyl-3[(1,2-dihydro-2-oxoquinoxalin-3-yl)methyl]-1H-pyrazolo[4,3-c] quinolin- 4(5H)-one (13).}

A mixture of compound $\mathbf{1 1}(0.50 \mathrm{~g}, 1.30 \mathrm{mmol})$ and hydrazine hydrate $(0.06 \mathrm{~mL})$, in absolute ethanol $(20 \mathrm{~mL})$, containing one drop of sulfuric acid, was heated under reflux for $10 \mathrm{~h}$. The solid obtained during heating was filtered and crystallized from DMF to give compound 13 as dark orange crystals, yield $(0.30 \mathrm{~g}, 61 \%)$, m.p. > $300{ }^{\circ} \mathrm{C}$; FT-IR $(\mathrm{KBr}) \mathrm{v}_{\max }$ 3196, $3129(\mathrm{NH}), 3040\left(\mathrm{CH}_{\text {arom. }}\right), 2975\left(\mathrm{CH}_{\text {aliph. }}\right), 1685\left(\mathrm{C}=\mathrm{O}_{\text {amide }}\right), 1647\left(\mathrm{C}=\mathrm{O}_{\text {quinolone }}\right), 1596$ $(\mathrm{C}=\mathrm{N})$ and $1559 \mathrm{~cm}^{-1}(\mathrm{C}=\mathrm{C}) ;{ }^{1} \mathrm{H} \mathrm{NMR}\left(\mathrm{DMSO}_{-} \mathrm{d}_{6}, 300 \mathrm{MHz}\right) \delta 1.26(\mathrm{t}, 3 \mathrm{H}, \mathrm{J}=6.9 \mathrm{~Hz}$, $\left.\mathrm{CH}_{2} \mathrm{CH}_{3}\right), 2.60\left(\mathrm{~s}, 2 \mathrm{H}, \mathrm{CH}_{2}\right), 4.28\left(\mathrm{q}, 2 \mathrm{H}, \mathrm{J}=6.9 \mathrm{~Hz}, \mathrm{CH}_{2} \mathrm{CH}_{3}\right), 7.01-8.20(\mathrm{~m}, 8 \mathrm{H}, \mathrm{Ar}-\mathrm{H})$, 12.09 (bs, $1 \mathrm{H}, \mathrm{NH}$ exchangeable with $\mathrm{D}_{2} \mathrm{O}$ ), 13.68 (bs, $1 \mathrm{H}, \mathrm{NH}$ exchangeable with $\mathrm{D}_{2} \mathrm{O}$ ); Mass spectrum, m/z (rel. int. \%): $370\left[\mathrm{M}^{+}-1\right]$ (0.4), 347 (6), 319 (2), 291 (2), 216 (24), 189 (9), $188(14), 160(42), 132(100), 120(30), 104(15)$ and 64 (16). Anal. Calcd for $\mathrm{C}_{21} \mathrm{H}_{17} \mathrm{~N}_{5} \mathrm{O}_{2}$ (371.40), C, 67.80\%; $\mathrm{H}, 4.69 \% ; \mathrm{N}, 18.98 \%$; found, $\mathrm{C}, 67.91 \% ; \mathrm{H}, 4.61 \%$; , $18.86 \%$.

\section{1-Ethyl-3-[2-chloro-2-(1,2-dihydro-2-oxoquinoxalin-3-yl)acetyl]-4- hydroxyquinolin-2(1H)-one (14).}

To a suspension of compound $11(0.50 \mathrm{~g}, 1.30 \mathrm{mmol}$ ) in 1,4-dioxane (30 mL), sulfuryl chloride $(3 \mathrm{~mL})$ was added, then the reaction mixture was stirred at room temperature for 1 $\mathrm{h}$ and poured onto crushed ice $(\mathrm{ca} .100 \mathrm{~g})$. The formed precipitate was collected by filtration and crystallized from EtOH to give compound 14 as pale yellow crystals, yield $(0.38 \mathrm{~g}$, 91\%). m.p. 240-241 ${ }^{\circ} \mathrm{C}$; FT-IR (KBr) $v_{\max } 3446(\mathrm{OH}), 3223(\mathrm{NH}), 3040\left(\mathrm{CH}_{\text {arom. }}\right), 2961$, $2925\left(\mathrm{CH}_{\text {aliph. }}\right), 1670\left(\mathrm{C}=\mathrm{O}_{\text {amide }}\right.$ and $\left.\mathrm{C}=\mathrm{O}_{\text {ketone }}\right), 1636\left(\mathrm{C}=\mathrm{O}_{\text {quinolone }}\right)$ and $1559 \mathrm{~cm}^{-1}(\mathrm{C}=\mathrm{N}$ and $\mathrm{C}=\mathrm{C}) ;{ }^{1} \mathrm{H}$ NMR (DMSO- $\left.d_{6}, 300 \mathrm{MHz}\right) \delta 1.26\left(\mathrm{t}, 3 \mathrm{H}, J=6.9 \mathrm{~Hz}, \mathrm{CH}_{2} \mathrm{CH}_{3}\right), 4.31(\mathrm{q}, 2 \mathrm{H}, \mathrm{J}=6.9$ $\left.\mathrm{Hz}, \mathrm{CH}_{2} \mathrm{CH}_{3}\right), 7.03(\mathrm{~s}, \mathrm{H}, \mathrm{CHCl}), 7.20-7.25(\mathrm{~m}, 2 \mathrm{H}, \mathrm{Ar}-\mathrm{H}), 7.31-7.37(\mathrm{~m}, 2 \mathrm{H}, \mathrm{Ar}-\mathrm{H}), 7.48(\mathrm{~d}$, $1 \mathrm{H}, \mathrm{J}=8.7 \mathrm{~Hz}, \mathrm{Ar}-\mathrm{H}), 7.63(\mathrm{t}, 1 \mathrm{H}, J=6.9 \mathrm{~Hz}, \mathrm{Ar}-\mathrm{H}), 7.79(\mathrm{~d}, 1 \mathrm{H}, J=6.9 \mathrm{~Hz}, \mathrm{Ar}-\mathrm{H}), 8.23(\mathrm{~d}$, $1 \mathrm{H}, J=6.9 \mathrm{~Hz}, \mathrm{Ar}-\mathrm{H}), 13.69$ (bs, $1 \mathrm{H}, \mathrm{NH}$ exchangeable with $\left.\mathrm{D}_{2} \mathrm{O}\right)$. Mass spectrum, $\mathrm{m} / \mathrm{z}\left(\mathrm{I}_{\mathrm{r}}\right.$ \%): $410\left(\mathrm{M}^{+}, 4\right), 375$ (3), 347 (6), 305 (49), 290 (20), 277 (42), 248 (17), 213 (3), 189 (6), 172 (5), 160 (6), $144(10), 132(42), 118(21), 91$ (24), 77 (75) and 64 (100). Anal. Calcd for $\mathrm{C}_{21} \mathrm{H}_{16} \mathrm{ClN}_{3} \mathrm{O}_{4}$ (409.83), C, $61.50 \% ; \mathrm{H}, 4.00 \%$;, $10.44 \%$; found, $\mathrm{C}, 61.55 \% ; \mathrm{H}$, $3.94 \%$; N , $10.25 \%$. 


\section{1-Ethyl-3-[2-dibromo-2-(1,2-dihydro-2-oxoquinoxalin-3-yl)acetyl]-4- hydroxyquinolin-2(1H)-one (15).}

A solution of bromine $(0.07 \mathrm{~mL})$ in acetic acid $(10 \mathrm{~mL})$ was added dropwise to a solution of compound $11(0.50 \mathrm{~g}, 1.30 \mathrm{mmol})$ in acetic acid $(10 \mathrm{~mL})$. The reaction mixture was stirring at room temperature for $30 \mathrm{~min}$. The orange crystals obtained during stirring were filtered and recrystallized from ethanol to give 15 as orange-red crystals, yield $(0.30 \mathrm{~g}, 49 \%)$, mp > 300 ○ C; FT-IR ( KBr, cm $\left.{ }^{-1}\right): 3445(\mathrm{OH}), 3201(\mathrm{NH}), 3090\left(\mathrm{CH}_{\text {arom. }}\right), 2923,2853\left(\mathrm{CH}_{\text {aliph. }}\right)$, $1700\left(\mathrm{C}=\mathrm{O}_{\text {ketone }}\right), 1652\left(\mathrm{C}=\mathrm{O}_{\text {amide }}\right), 1640\left(\mathrm{C}=\mathrm{O}_{\text {quinolone }}\right), 1615(\mathrm{C}=\mathrm{N})$ and $1575(\mathrm{C}=\mathrm{C}) ;{ }^{1} \mathrm{H}$ NMR (DMSO-d $\left.d_{6}, 300 \mathrm{MHz}\right) \delta 1.29\left(\mathrm{t}, 3 \mathrm{H}, J=6.9 \mathrm{~Hz}, \mathrm{CH}_{2} \mathrm{CH}_{3}\right), 4.31(\mathrm{q}, 2 \mathrm{H}, J=6.9 \mathrm{~Hz}$, $\left.\mathrm{CH}_{2} \mathrm{CH}_{3}\right), 7.05(\mathrm{~s}, \mathrm{H}, \mathrm{CHBr}), 7.29-7.99(\mathrm{~m}, 7 \mathrm{H}, \mathrm{Ar}-\mathrm{H}), 8.29(\mathrm{t}, 1 \mathrm{H}, \mathrm{J}=8.7 \mathrm{~Hz}, \mathrm{Ar}-\mathrm{H}), 12.9$ (bs, $1 \mathrm{H}, \mathrm{NH}$ exchangeable with $\left.\mathrm{D}_{2} \mathrm{O}\right)$; Mass spectrum, $\mathrm{m} / \mathrm{z}$ (rel. int. \%): $454\left(\mathrm{M}^{+\cdot}\right](5), 413$ (4), 411 (4), 360 (4), 358 (5), 288 (4), 286 (4), 227 (3), 225 (4), 216 (4), 187 (10), 155 (4), 153 (4), 136 (4), 134 (4), 121 (5), 104 (7), 80 (100) and 77 (8). Anal. Calcd for $\mathrm{C}_{21} \mathrm{H}_{16} \mathrm{BrN}_{3} \mathrm{O}_{4}$ (454.28), C, 55.78\%; $\mathrm{H}, 3.67 \%$; N, 9.34\%; found, C, 55.52\%; $\mathrm{H}, 3.55 \%$; $\mathrm{N}$, $9.25 \%$.

\section{1-Ethyl-3-[2-nitro-2-(1,2-dihydro-2-oxoquinoxalin-3-yl)acetyl]-4-hydroxyquinolin- 2(1H)-one (16).}

To solution of compound $11(1.00 \mathrm{~g}, 2.60 \mathrm{mmol})$ in glacial acetic acid $(10 \mathrm{~mL})$, a mixture of concentrated nitric acid $(2 \mathrm{~mL})$ and concentrated sulfuric acid $(2 \mathrm{~mL})$ was carefully added portion wise with continuous stirring at room temperature for $30 \mathrm{~min}$ then poured onto crushed ice (ca. $50 \mathrm{gm})$. The solid obtained was filtered, washed with water, air-dried and crystallized from EtOH to give compound $\mathbf{1 6}$ as yellow crystals, yield $(0.50 \mathrm{~g}, 45 \%)$, m.p. $270{ }^{\circ} \mathrm{C}$. FT-IR $\left(\mathrm{KBr}_{\mathrm{cm}} \mathrm{cm}^{-1}\right): 3435(\mathrm{OH}), 3223(\mathrm{NH}), 2923,2854\left(\mathrm{CH}_{\text {aliph. }}\right), 1751\left(\mathrm{C}=\mathrm{O}_{\text {ketone }}\right)$, $1676\left(\mathrm{C}=\mathrm{O}_{\text {amide }}\right), 1639$ ( $\left.\mathrm{C}=\mathrm{O}_{\text {quinolone }}\right)$ and $1579(\mathrm{C}=\mathrm{N}$ and $\mathrm{C}=\mathrm{C}) .{ }^{1} \mathrm{H}$ NMR (DMSO- $d_{6}, \delta$, $300 \mathrm{MHz}): 1.22\left(\mathrm{t}, 3 \mathrm{H}, \mathrm{CH}_{2} \mathrm{CH}_{3}\right), 4.48\left(\mathrm{q}, 2 \mathrm{H}, \mathrm{CH}_{2} \mathrm{CH}_{3}\right), 7.07\left(\mathrm{~s}, 1 \mathrm{H}, \mathrm{CHNO}_{2}\right), 7.42-8.22(\mathrm{~m}$, $8 \mathrm{H}, \mathrm{Ar}-\mathrm{H}), 13.2$ (bs, $1 \mathrm{H}, \mathrm{NH}$ exchangeable with $\left.\mathrm{D}_{2} \mathrm{O}\right)$. Mass spectrum, $\mathrm{m} / \mathrm{z}\left(\mathrm{I}_{\mathrm{r}} \%\right): 420\left(\mathrm{M}^{+}\right.$, 1), 305 (1), 267 (1), 215 (2), 188 (2), 155 (3), 149 (4), 139 (5), 132 (3), 125 (11), 121 (11), 85 (36), $77(6), 64$ (30) and 57 (100). Anal. Calcd. for $\mathrm{C}_{21} \mathrm{H}_{16} \mathrm{~N}_{4} \mathrm{O}_{6}(420.38), \mathrm{C}$, $60.00 \% ; H, 3.84 \% ; N, 13.33 \%$; found, $C, 60.50 \% ; H, 4.09 \% ; N, 13.58 \%$.

\section{1-Ethyl-3-[2-(2,3,4,5-tetrahydro-3-thioxo-4-amino-5-oxo-1,2,4-triazin-6- yl)acetyl]-4-hydroxyquinolin-2(1H)-one (17).}

A mixture of diketoester $1(0.50 \mathrm{~g}, 1.51 \mathrm{mmol})$ and thiocarbohydrazide $(0.106 \mathrm{~g}, 1.51$ $\mathrm{mmol})$, in absolute ethanol $(20 \mathrm{~mL})$, containing few drops of acetic acid, was heated under reflux for $2 \mathrm{~h}$. The orange crystals obtained during heating was filtered and crystallized from DMF to give compound 17 as pale orange crytals, yield $(0.56 \mathrm{~g}, 57 \%)$, m.p. $>300{ }^{\circ} \mathrm{C}$; FT-IR $(\mathrm{KBr}) \mathrm{v}_{\max } 3464(\mathrm{OH}), 3333(\mathrm{NH}), 3238\left(\mathrm{NH}_{2}\right), 2981\left(\mathrm{CH}_{\text {aliph. }}\right), 1669\left(\mathrm{C}=\mathrm{O}_{\text {triazine }}\right), 1646$ $\left(\mathrm{C}=\mathrm{O}_{\text {ketone }}\right), 1621\left(\mathrm{C}=\mathrm{O}_{\text {quinolone }}\right), 1595(\mathrm{C}=\mathrm{N})$ and $1563 \mathrm{~cm}^{-1}(\mathrm{C}=\mathrm{C}) ;{ }^{1} \mathrm{H}$ NMR (DMSO- $d_{6}, 300$ $\mathrm{MHz}) \delta 1.20\left(\mathrm{t}, 3 \mathrm{H}, J=6.9 \mathrm{~Hz}, \mathrm{CH}_{2} \mathrm{CH}_{3}\right), 4.25\left(\mathrm{q}, 2 \mathrm{H}, J=6.9 \mathrm{~Hz}, \mathrm{CH}_{2} \mathrm{CH}_{3}\right), 4.48\left(\mathrm{~s}, 2 \mathrm{H}, \mathrm{CH}_{2}\right)$, 6.54 (bs, $2 \mathrm{H}, \mathrm{NH}_{2}$ exchangeable with $\left.\mathrm{D}_{2} \mathrm{O}\right), 7.34\left(\mathrm{t}, 1 \mathrm{H}, J=7.2 \mathrm{~Hz}, \mathrm{H}-6_{\text {quinolone }}\right), 7.61(\mathrm{~d}, 1 \mathrm{H}$, $\left.\mathrm{J}=8.7 \mathrm{~Hz}, \mathrm{H}-8_{\text {quinolone }}\right), 7.80\left(\mathrm{t}, 1 \mathrm{H}, \mathrm{J}=7.5 \mathrm{~Hz}, \mathrm{H}-7_{\text {quinolone }}\right), 8.14(\mathrm{~d}, 1 \mathrm{H}, \mathrm{J}=8.1 \mathrm{~Hz}, \mathrm{H}-$ $5_{\text {quinolone }}$ ), 12.70 (bs, $1 \mathrm{H}, \mathrm{NH}$ exchangeable with $\mathrm{D}_{2} \mathrm{O}$ ), 14.00 (bs, $1 \mathrm{H}, \mathrm{OH}$ exchangeable with $\mathrm{D}_{2} \mathrm{O}$ ); mass spectrum, $\mathrm{m} / \mathrm{z}$ (rel. int. \%): $373\left[\mathrm{M}^{+} \cdot\right]$ (17), 357 (11), 327 (1), 267 (2), 255 (6), 229 (7), 216 (100), 189 (8), 161 (3), $132(14), 119(6), 103$ (5) and 64 (10). Anal. Calcd for $\mathrm{C}_{16} \mathrm{H}_{15} \mathrm{~N}_{5} \mathrm{O}_{4} \mathrm{~S}$ (373.39), C, $51.50 \% ; \mathrm{H}, 4.19 \% ; \mathrm{N}, 18.68 \%$; S, $8.65 \%$; found, C, $51.47 \% ; \mathrm{H}, 4.05 \% ; \mathrm{N}, 18.76 \% ; \mathrm{S}, 8.59 \%$. 
N-\{6-[2-(1-ethyl-4-hydroxy-2-oxo-1,2-dihydroquinolin-3-yl)-2-oxoethyl]-5-oxo-3thioxo-2,5-dihydro-1,2,4-triazin-4(3H)-yl\}acetamide (18).

A solution of compound $17(0.5 \mathrm{~g}, 1.20 \mathrm{mmol})$ in acetic acid $(10 \mathrm{~mL})$ was heated under reflux for $4 \mathrm{~h}$. The solid obtained during heating was filtered and crystallized from DMF to give compound 18 as orange crystals, yield $(0.25 \mathrm{~g}, 45 \%)$, m.p. $>300{ }^{\circ} \mathrm{C}$; FT-IR $(\mathrm{KBr}) \mathrm{v}_{\max }$ $3433(\mathrm{OH}), 3201(\mathrm{NH}), 3238\left(\mathrm{NH}_{2}\right), 2924$ and $2856\left(\mathrm{CH}_{\text {aliph. }}\right), 1739\left(\mathrm{C}=\mathrm{O}_{\text {acetamide }}\right), 1675$ $\left(\mathrm{C}=\mathrm{O}_{\text {triazine }}\right), 1626\left(\mathrm{C}=\mathrm{O}_{\text {quinolone }}\right.$ and $\left.\mathrm{C}=\mathrm{O}_{\text {ketone }}\right), 1595(\mathrm{C}=\mathrm{N})$ and $1500 \mathrm{~cm}^{-1}(\mathrm{C}=\mathrm{C}) ;{ }^{1} \mathrm{H}$ NMR (DMSO-d $\left.{ }_{6}, 300 \mathrm{MHz}\right) \delta 1.30\left(\mathrm{t}, 3 \mathrm{H}, \mathrm{CH}_{2} \mathrm{CH}_{3}\right), 3.09\left(\mathrm{~s}, 3 \mathrm{H}, \mathrm{CH}_{3}\right), 4.59\left(\mathrm{q}, 2 \mathrm{H}, \mathrm{CH}_{2} \mathrm{CH}_{3}\right), 4.29$ $(\mathrm{s}, 2 \mathrm{H}, \mathrm{CH} 2), 7.53\left(\mathrm{t}, 1 \mathrm{H}, \mathrm{H}-6_{\text {quinolone }}\right), 7.81-7.86\left(\mathrm{~m}, 2 \mathrm{H}, \mathrm{H}-8_{\text {quinolone }}\right.$ and $\left.\mathrm{H}-7_{\text {quinolone }}\right), 8.22$ (d, $1 \mathrm{H}, \mathrm{H}-5_{\text {quinolone }}$ ), 8.97 (bs, $1 \mathrm{H}, \mathrm{NH}$ exchangeable with $\mathrm{D}_{2} \mathrm{O}$ ), 12.7 (bs, $1 \mathrm{H}, \mathrm{NH}$ exchangeable with $\left.\mathrm{D}_{2} \mathrm{O}\right)$. Mass spectrum, $\mathrm{m} / \mathrm{z}\left(\mathrm{I}_{\mathrm{r}} \%\right)$ : $415\left(\mathrm{M}^{+}, 2\right), 389(4), 376(26), 332$ (3), 305 (26), 277 (53), 215 (2), 189 (3), 169 (4), 132 (5), 119 (4), 101 (7) 91 (5), 77 (9) and 64 (100). Anal. Calcd for $\mathrm{C}_{18} \mathrm{H}_{17} \mathrm{~N}_{5} \mathrm{O}_{5} \mathrm{~S}$ (415.42), C, 52.12\%; H, 4.22\%; N, 16.78\%; S, $7.68 \%$; found, $\mathrm{C}, 52.04 \% ; \mathrm{H}, 4.12 \% ; \mathrm{N}, 16.86 \% ; \mathrm{S}, 7.72 \%$.

\section{Ethyl 3-cyano-6-(1-ethyl-1,2-dihydro-4-hydroxy-2-oxoquinolin-3-yl)-2-oxo-2H- pyran-4-carboxylate (19).}

A mixture of diketoester $3(0.50 \mathrm{~g}, 1.51 \mathrm{mmol})$ and malononitrile $(0.26 \mathrm{~g}, 1.51 \mathrm{mmol})$ in ethanol $(20 \mathrm{~mL})$ containing few drops of TEA or acetic acid was heated under reflux for 30 min. The solid that formed during heating was filtered and crystallized from DMF to give compound 19 as pale brown crystal, yield $(0.30 \mathrm{~g}, 41 \%)$, m.p. 233-234 ${ }^{\circ} \mathrm{C}$; FT-IR (KBr) $\mathrm{v}_{\max } 3445(\mathrm{OH}), 3265(\mathrm{NH}), 2985,2922,2843\left(\mathrm{CH}_{\text {aliph. }}\right), 2228(\mathrm{C} \equiv \mathrm{N}), 1726\left(\mathrm{C}=\mathrm{O}_{\text {ester }}\right), 1634$ $\left(\mathrm{C}=\mathrm{O}_{\text {quinolone }}\right), 1595(\mathrm{C}=\mathrm{N})$ and $1571 \mathrm{~cm}^{-1}(\mathrm{C}=\mathrm{C}) .{ }^{1} \mathrm{H} \mathrm{NMR}\left(\mathrm{DMSO}-d_{6}, 300 \mathrm{MHz}\right) \delta 1.24(\mathrm{t}$, $\left.3 \mathrm{H}, J=6.9 \mathrm{~Hz}, \mathrm{~N}-\mathrm{CH}_{2} \mathrm{CH}_{3}\right), 1.49\left(\mathrm{t}, 3 \mathrm{H}, J=6.9 \mathrm{~Hz}, \mathrm{O}-\mathrm{CH}_{2} \mathrm{CH}_{3}\right), 4.32(\mathrm{q}, 2 \mathrm{H}, J=6.9 \mathrm{~Hz}, \mathrm{~N}-$ $\left.\mathrm{CH}_{2} \mathrm{CH}_{3}\right), 4.49\left(\mathrm{q}, 2 \mathrm{H}, \mathrm{J}=6.9 \mathrm{~Hz}, \mathrm{O}-\mathrm{CH}_{2} \mathrm{CH}_{3}\right), 7.29(\mathrm{t}, 1 \mathrm{H}, \mathrm{J}=7.2 \mathrm{~Hz}, \mathrm{H}-6), 7.56(\mathrm{~d}, 1 \mathrm{H}, \mathrm{J}=8.4$ $\mathrm{Hz}, \mathrm{H}-8), 7.73(\mathrm{t}, 1 \mathrm{H}, J=8.4 \mathrm{~Hz}, \mathrm{H}-7), 8.08(\mathrm{~d}, 1 \mathrm{H}, J=8.1 \mathrm{~Hz}, \mathrm{H}-5), 8.20\left(\mathrm{~s}, 1 \mathrm{H}, \mathrm{H}-5_{\text {pyrane }}\right)$, 9.29 (bs, $1 \mathrm{H}, \mathrm{NH}$ exchangeable with $\mathrm{D}_{2} \mathrm{O}$ ), 14 (bs, $1 \mathrm{H}, \mathrm{OH}$ exchangeable with $\mathrm{D}_{2} \mathrm{O}$ ). Mass spectrum, m/z (rel. int. \%): 379 [M+.] (14), 365 (15), 351 (29.54), 323 (12.8), 278 (6.9), 160 (22), 146 (66.86), 132 (100), 120 (27.5), 104 (9.3) and 77 (5.35). Anal. Calcd for $\mathrm{C}_{20} \mathrm{H}_{17} \mathrm{~N}_{3} \mathrm{O}_{5}$ (379.38), C, 63.20\%; $\mathrm{H}, 4.61 \% ; \mathrm{N}, 11.00 \%$; found, $\mathrm{C}, 63.32 \% ; \mathrm{H}, 4.52 \%$; , $11.08 \%$.

\section{RESULTS AND DISCUSSION}

Earlier Ismail and Mohamed have reported the synthesis of ethyl 4-(1-ethyl-4-hydroxy-2oxo-1,2-dihydroquinolin-3-yl)-2,4-dioxobutyrate (1), in good yield [15]. The chemical behavior of this diketoester $\mathbf{1}$ was studied towards a variety of 1,2- binucleophiles, such as cyanoacetohydrazide, nicotinohydrazide and 7-chloroquinolin-4-ylhydrazine (2). Thus, treatment of diketoester $\mathbf{1}$ with these hydrazine derivatives, in absolute ethanol containing few drops of acetic acid, gave the corresponding pyrazole derivatives 3-5, in $37-54 \%$ yields (Scheme 1). Formation of pyrazole derivatives 3-5 may occur initially via condensation of $\mathrm{NH}_{2}$ group at $\left(\mathrm{C}=\mathrm{O}_{\mathrm{a}-\mathrm{keto}}\right)$ group to give hydrazone intermediates which in turn underwent an intramolecular cyclocondensation at $\left(\mathrm{C}=\mathrm{O}_{y-\text { keto }}\right)$ group. The FT-IR spectra of pyrazoles 3-5 displayed characteristic absorption bands attributed to $\mathrm{C}=\mathrm{O}_{\text {ester }}$ at 1756,1706 , and 1707 $\mathrm{cm}^{-1}$, respectively. 
The FT-IR spectrum of compound $\mathbf{3}$ showed characteristic absorption bands attributed to the $\mathrm{C} \equiv \mathrm{N}$ and $\mathrm{C}=\mathrm{O}_{\text {ketone }}$ at 2263 and $1695 \mathrm{~cm}^{-1}$, respectively. The ${ }^{1} \mathrm{H}$ NMR spectra of compounds 3-5 showed characteristic signals singlet attributed to $\mathrm{H}-4_{\text {pyrazole }}$ at $\delta 7.20,7.10$ and 6.40 ppm, respectively. Furthermore, the mass spectra of compounds $\mathbf{3}, \mathbf{4}$ and $\mathbf{5}$ showed their molecular ion peaks at $\mathrm{m} / \mathrm{z}$ 394, 432 and 489 and assigned their formula weights, respectively.

Next, the diketoester $\mathbf{1}$ was allowed to react with some 1,3-N,N-binucleophiles. Condensation of diketoester 1 with 3 -amino[1,2,4]triazole (6), in boiling ethanol containing few drops of acetic acid, led to [1,2,4]triazolo[4,3-a]pyrimidine derivative $\mathbf{7}$ bearing the quinoline moiety (Scheme 2). The FT-IR spectrum of compound $\mathbf{7}$ showed characteristic absorption vibrations at $3447(\mathrm{O}-\mathrm{H}), 1751\left(\mathrm{C}=\mathrm{O}_{\text {ester }}\right)$ and $1635 \mathrm{~cm}^{-1}\left(\mathrm{C}=\mathrm{O}_{\text {quinoline }}\right)$. Its ${ }^{1} \mathrm{H}$ NMR spectrum showed two characteristic singlets at $\delta 8.73\left(\mathrm{H}-5_{\text {pyrimidine }}\right)$ and $9.40 \mathrm{ppm}(\mathrm{H}-$ $3_{\text {triazole }}$ ). Further, the mass spectrum of compound $\mathbf{7}$ showed the molecular ion peak at $\mathrm{m} / \mathrm{z}$ 379 , which is coincident with its formula weight (379.39) and supports the identity of the structure.

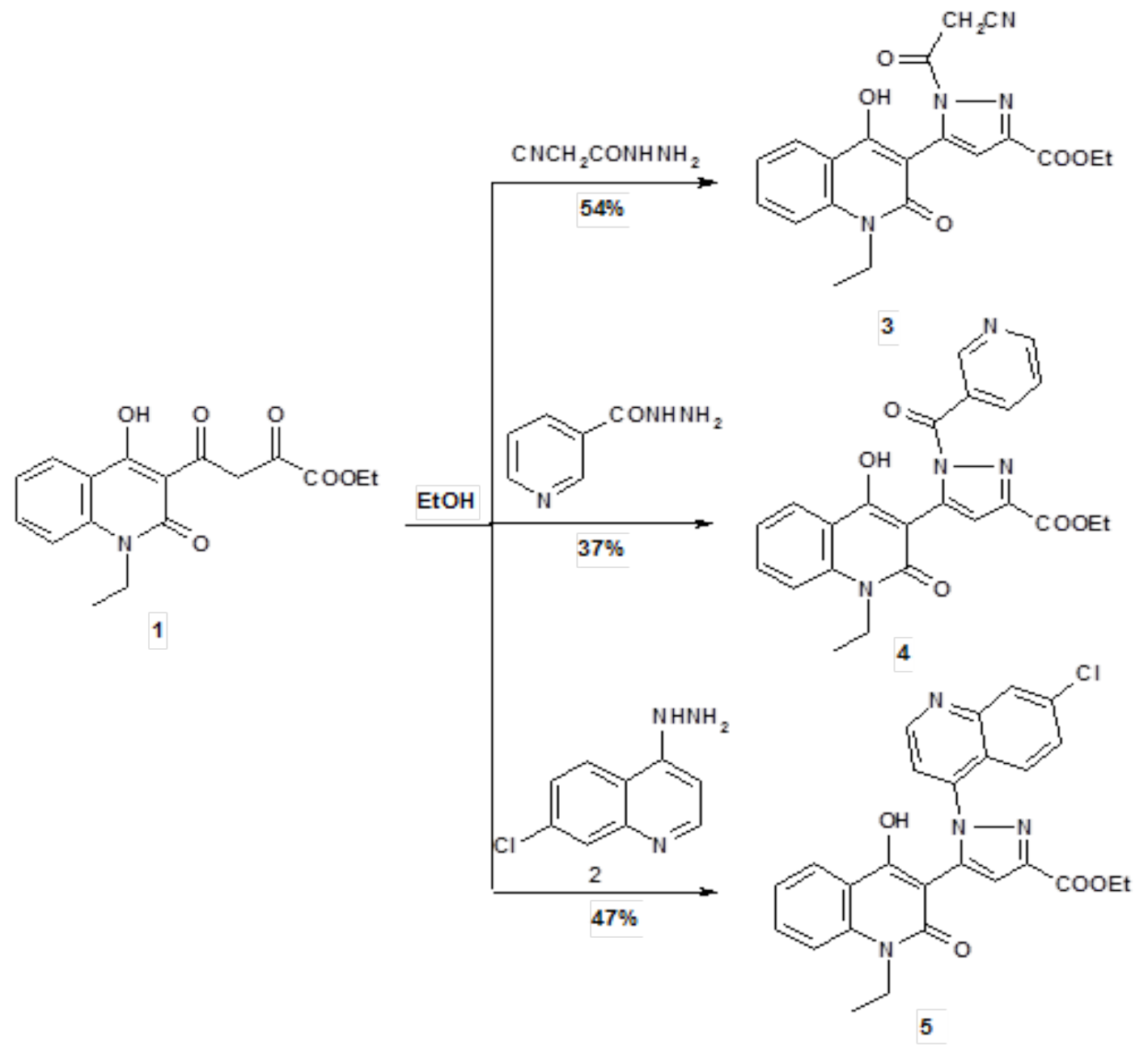

Scheme 1. 


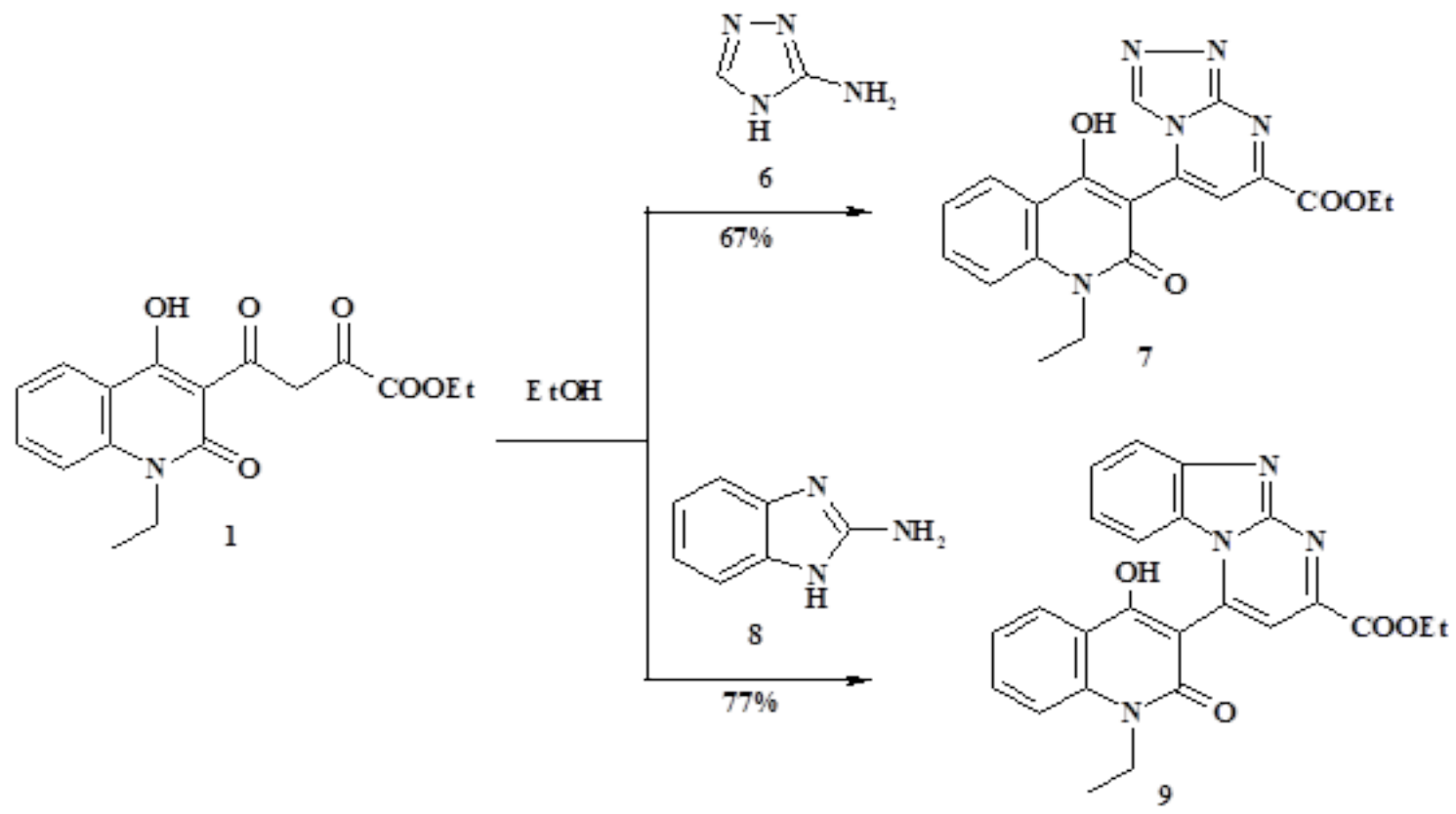

Scheme 2.

In the same manner, condensation of diketoester $\mathbf{1}$ with 2-aminobenzimidazole (8), in absolute ethanol containing few drops of acetic acid, produced pyrimido[1,2-a] benzimidazole derivative 9 linked to the quinolinone moiety (Scheme 2). The FT-IR spectrum indicated characteristic absorption vibrations at $3359(\mathrm{O}-\mathrm{H}), 1707\left(\mathrm{C}=\mathrm{O}_{\text {ester }}\right)$ and $1645 \mathrm{~cm}^{-1}\left(\mathrm{C}=\mathrm{O}_{\text {quinolone }}\right)$. The ${ }^{1} \mathrm{H}$ NMR spectrum of compound 9 showed characteristic singlet at $\delta 6.90\left(\mathrm{H}-5_{\text {pyrimidine }}\right)$. Further, its mass spectrum showed the molecular ion peak at $\mathrm{m} / \mathrm{z} 370$ and confirms the suggested structure.

Then, the chemical reactivity of diketoester $\mathbf{1}$ was studied towards a variety of 1,4binucleophiles. Therefore, treatment of diketoester $\mathbf{1}$ with ethylene diamine, 1,2-phenylene diamine and 2-aminophenol, in boiling ethanol containing few drops of acetic acid, furnished the corresponding pyrazine, quinoxaline and benzoxazine derivatives 10-12, respectively (Scheme 3).

The ${ }^{1} \mathrm{H}$ NMR spectra of compounds 10, 11 and 12 showed a singlet distinguishable for the active methylene protons at $\delta 2.70$ (in compound 10) and $2.60 \mathrm{ppm}$ (in compound $\mathbf{1 1}$ and 12). In addition, the FT-IR spectra of compounds $\mathbf{1 0}$ and $\mathbf{1 1}$ demonstrated stretching vibrations attributed to $\left(\mathrm{C}=\mathrm{O}_{\text {amide }}\right)$ at 1675 and $1685 \mathrm{~cm}^{-1}$, respectively, while the FT-IR spectrum of compound $\mathbf{1 2}$ displayed characteristic absorption band assigned to $\left(\mathrm{C}=\mathrm{O}_{\mathrm{a}-}\right.$ pyrone) group at $1752 \mathrm{~cm}^{-1}$. 


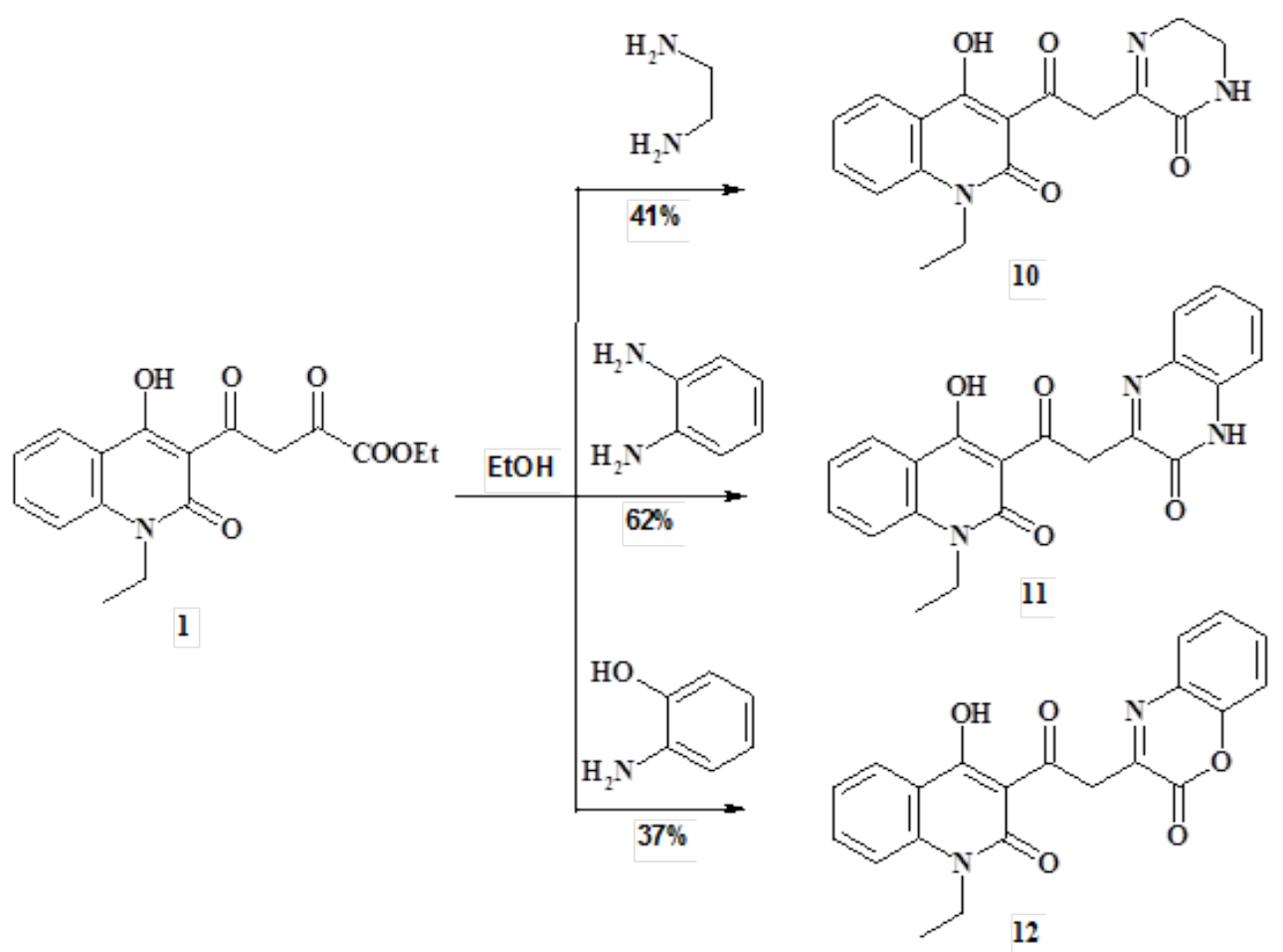

Scheme 3.

Condensation of compound $\mathbf{1 1}$ with hydrazine hydrate in boiling ethanol containing few drops of sulfuric acid produced pyrazolo[4,3-c]quinolinone 13 linked with a quinoxaline moiety in one molecular frame through an active methylene group (Scheme 4 ). The ${ }^{1} \mathrm{H}$ NMR spectrum of compound $\mathbf{1 3}$ showed a singlet distinguishable for the active methylene protons 2.60 and showed exchangeable signals at $\delta 12.09$ and 13.68 assigned to two NH protons.<smiles>CCn1c(=O)c(C(=O)Cc2nc3ccccc3[nH]c2=O)c(O)c2ccccc21</smiles><smiles>CCCCC(=O)NN</smiles><smiles>CCn1c(=O)c2c(Cc3nc4ccccc4[nH]c3=O)n[nH]c2c2ccccc21</smiles>

Scheme 4.

Next, the chemical reactivity of compound $\mathbf{1 1}$ was studied towards some electrophilic reagents. Chlorination, bromination and nitration of compound $\mathbf{1 1}$ afforded the chlorinated, brominated and nitrated products 14-16, respectively (Scheme 5). The ${ }^{1} \mathrm{H}$ NMR spectra of compounds 14, 15 and $\mathbf{1 6}$ showed characteristic singlets attributed to the $\mathrm{CH}_{\text {aliphatic }}$ protons at $7.03,7.05$ and 7.07 ppm, respectively. 


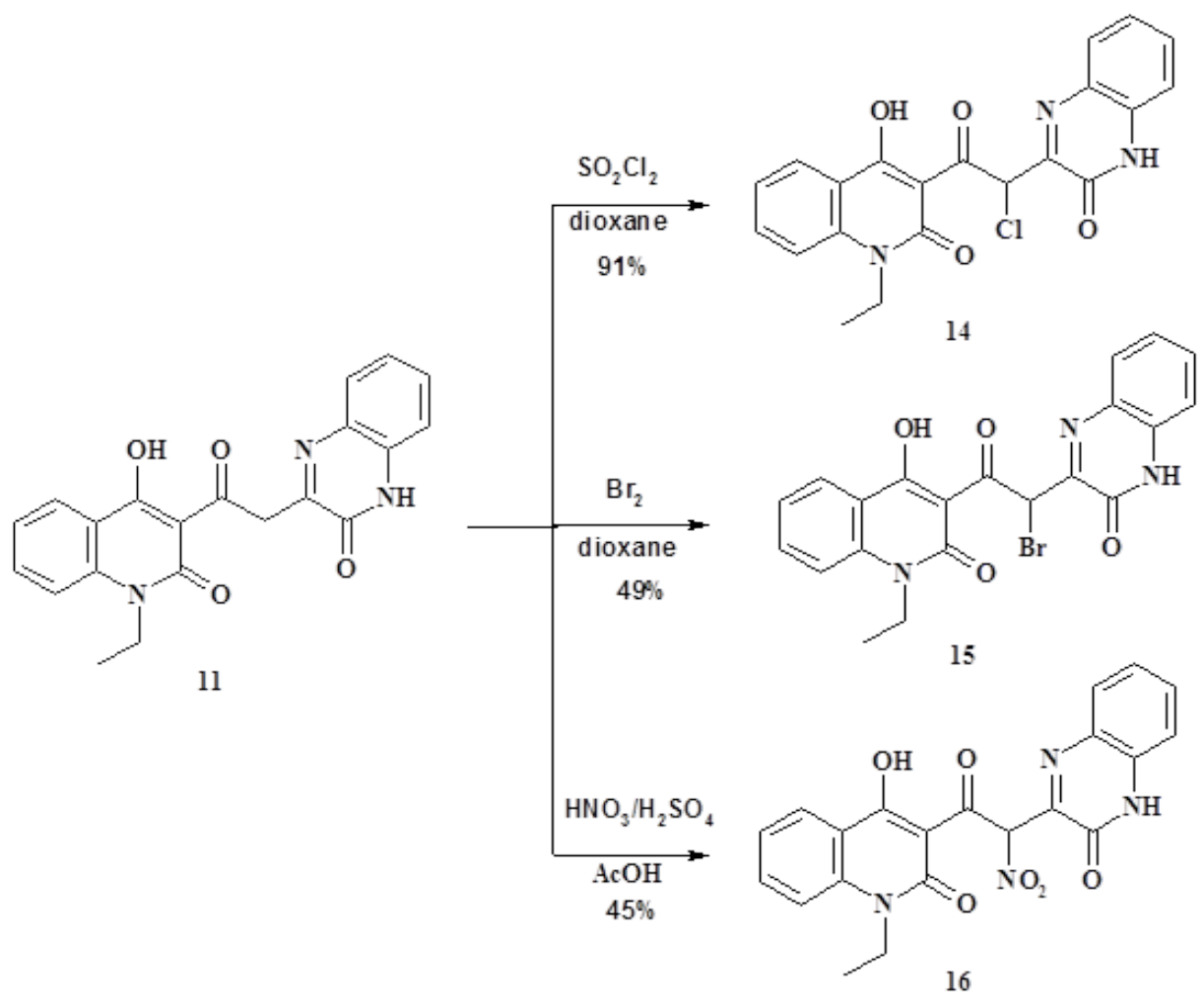

Scheme 5.

Treatment of diketoester $\mathbf{1}$ with thiocarbohydrazide, in absolute ethanol, containing few drops of acetic acid, furnished 1,2,4-triazine derivative 17 (Scheme 6). The FT-IR spectrum of compound 17 presented characteristic absorption bands at 3333, $3238\left(\mathrm{NH}, \mathrm{NH}_{2}\right)$ and $1669 \mathrm{~cm}^{-1}\left(\mathrm{C}=\mathrm{O}_{\text {triazine }}\right)$. The ${ }^{1} \mathrm{H}-\mathrm{NMR}$ spectrum of compound 17 showed a singlet assigned to $\mathrm{CH}_{2}$ protons at $\delta 4.48$, in addition to $\mathrm{D}_{2} \mathrm{O}$ exchangeable signals at $\delta 6.54$ and 12.70 assigned to $\mathrm{NH}_{2}$ and $\mathrm{NH}$ protons, respectively.

Acetylation of compound $\mathbf{1 7}$ by using glacial acetic acid under reflux led to the acetylated product 18 (Scheme 6). A new absorption band appeared at $1739 \mathrm{~cm}^{-1}$ in the FT-IR spectrum of compound 18, which might be assigned to $\left(\mathrm{C}=\mathrm{O}_{\text {acetamide }}\right)$. The ${ }^{1} \mathrm{H}$ NMR spectrum of compound $\mathbf{1 8}$ showed exchangeable signals at $\delta 8.97$ and 12.70 due to $2 \mathrm{NH}$, respectively.<smiles>CCn1c(=O)c(CC(=O)c2c(O)c3ccccc3n(CC)c2=O)c(O)c2ccccc21</smiles>

Scheme 6. 
Moreover, the present work aimed to study the chemical reactivity of diketoester $\mathbf{1}$ towards malononitrile as carbon nucleophilic reagents. Thus, condensation of diketoester $\mathbf{1}$ with malononitrile, in boiling ethanol containing some drops of acetic acid, led to 3-cyano-6-(1ethyl-1,2-dihydro-4-hydroxy-2-oxoquinolin-3-yl)-2-oxo- 2H-pyran-4-carboxylate (19) (Scheme 7). The FT-IR spectrum of compound 19 exhibited characteristic absorption bands at 2228 and $1726 \mathrm{~cm}^{-1}$ assigned to the $(\mathrm{C} \equiv \mathrm{N})$ and $\left(\mathrm{C}=\mathrm{O}_{\text {ester }}\right)$, respectively. Its ${ }^{1} \mathrm{H} \mathrm{NMR}$ spectrum indicated the presence of characteristic singlet at $\delta 8.20 \mathrm{ppm}$ due to $\mathrm{H}-5_{\text {pyrane. }}$.

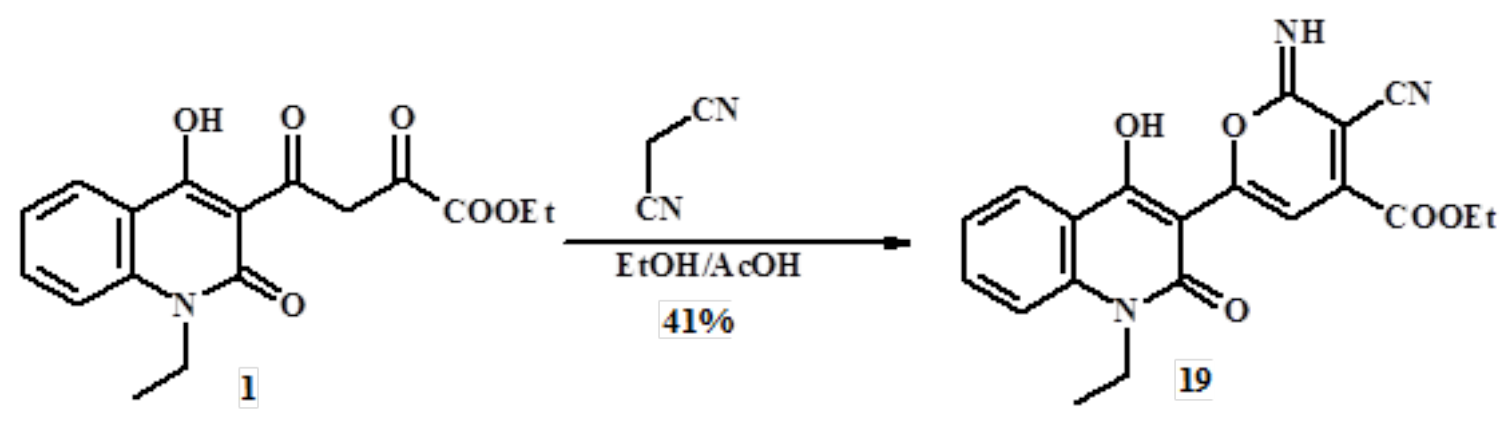

Scheme 7.

\section{REFERENCES}

[1] Ibrahim MA, Hassanin HM, Gabr YA, Alnamer YA. Studies on the chemical behavior of 3(nitroacetyl)-1-ethyl-4-hydroxyquinolin-2(1H)-one towards some electrophilic and nucleophilic reagents. Journal of the Brazilian Chemical Society. 2012 May;23(5):905-12. DOI: 10.1590/S010350532012000500016.

[2] Moradi Rufchahi EO, Pouramir H, Yazdanbakhsh MR, Yousefi H, Bagheri M, Rassa M. Novel azo dyes derived from 8-methyl-4-hydroxyl-2-quinolone: Synthesis, UV-vis studies and biological activity. Chinese Chemical Letters. 2013 May;24(5):425-8. DOI: 10.1016/j.cclet.2013.03.002.

[3] De Melo EB. Multivariate SAR/QSAR of 3-aryl-4-hydroxyquinolin-2(1H)-one derivatives as type I fatty acid synthase (FAS) inhibitors. European Journal of Medicinal Chemistry. 2010 Dec;45(12):5817-26. DOI: 10.1016/j.ejmech.2010.09.044.

[4] Abass M, Mostafa BB. Synthesis and evaluation of molluscicidal and larvicidal activities of some novel enaminones derived from 4-hydroxyquinolinones: Part IX. Bioorganic \& Medicinal Chemistry. 2005 Nov;13(22):6133-44. DOI: 10.1016/j.bmc.2005.06.038.

[5] Chen Y-L, Fang K-C, Sheu J-Y, Hsu S-L, Tzeng C-C. Synthesis and Antibacterial Evaluation of Certain Quinolone Derivatives. Journal of Medicinal Chemistry. 2001 Jul;44(14):2374-7. DOI: 10.1021/jm0100335.

[6] Detsi A, Bouloumbasi D, Prousis KC, Koufaki M, Athanasellis G, Melagraki G, et al. Design and Synthesis of Novel Quinolinone-3-aminoamides and Their a-Lipoic Acid Adducts as Antioxidant and Anti-inflammatory Agents. Journal of Medicinal Chemistry. 2007 May;50(10):2450-8. DOI:

10.1021/jm061173n. 
[7] Kaur K, Jain M, Kaur T, Jain R. Antimalarials from nature. Bioorganic \& Medicinal Chemistry. 2009 May;17(9):3229-56. DOI: 10.1016/j.bmc.2009.02.050.

[8] Behforouz M, Cai W, Mohammadi F, Stocksdale MG, Gu Z, Ahmadian M, et al. Synthesis and evaluation of antitumor activity of novel $\mathrm{N}$-acyllavendamycin analogues and quinoline-5,8-diones. Bioorganic \& Medicinal Chemistry. 2007 Jan 1;15(1):495-510. DOI: 10.1016/j.bmc.2006.09.039.

[9] Abas F, Lajis NH, Israf DA, Khozirah S, Umi Kalsom Y. Antioxidant and nitric oxide inhibition activities of selected Malay traditional vegetables. Food Chemistry. 2006 Apr;95(4):566-73. DOI: 10.1016/j.foodchem.2005.01.034.

[10] Rocha LG, Almeida JRGS, Macêdo RO, Barbosa-Filho JM. A review of natural products with antileishmanial activity. Phytomedicine. 2005 Jun;12(6-7):514-35. DOI:

10.1016/j.phymed.2003.10.006.

[11] Kuo R-Y, Chang F-R, Chen C-Y, Teng C-M, Yen H-F, Wu Y-C. Antiplatelet activity of Nmethoxycarbonyl aporphines from Rollinia mucosa. Phytochemistry. 2001 Jun;57(3):421-5. DOI: 10.1016/S0031-9422(01)00076-0.

[12] Chen Y-L, Chen I-L, Tzeng C-C, Wang T-C. Synthesis and Cytotoxicity Evaluation of CertainaMethylidene- $\gamma$-butyrolactones Bearing Coumarin, Flavone, Xanthone, Carbazole, and Dibenzofuran Moieties. Helvetica Chimica Acta. 2000 May 10;83(5):989-94. DOI: 10.1002/(SICI)15222675(20000510)83:5<989::AID-HLCA989>3.0.CO;2-E.

[13] Rivkin A, Kim YR, Goulet MT, Bays N, Hill AD, Kariv I, et al. 3-Aryl-4-hydroxyquinolin-2(1H)-one derivatives as type I fatty acid synthase inhibitors. Bioorganic \& Medicinal Chemistry Letters. 2006 Sep;16(17):4620-3. DOI: 10.1016/j.bmcl.2006.06.014.

[14] Roussaki M, Hall B, Lima SC, da Silva AC, Wilkinson S, Detsi A. Synthesis and anti-parasitic activity of a novel quinolinone-chalcone series. Bioorganic \& Medicinal Chemistry Letters. 2013 Dec;23(23):6436-41. DOI: 10.1016/j.bmcl.2013.09.047.

[15] Ismail M, Mohamed $H$. Synthesis and cyclization reactions with quinolinyl keto esters II. Synthesis of novel 3-diazolylquunolinones and their enzymic activity. Chemical Papers. 2005;59(2):127-38. URL: http://www.chempap.org/file_access.php?file=592a127.pdf. 


\section{4-(1-ETİL-1, 2-DİHİDRO-4-HİDROKSİ-2-OKSOKİNOLİN-3-İL)2,4-} DİOKSOBUTANOATIN BAZI NÜKLEOFİLİK REAKTİFLERE KARŞI KİMYASAL REAKTIVITESİ

Kinolin-2-on ile bağlanmış yeni bir seri heterosiklik sistemler, etil 4-(1-etil-1,2-dihidro-4hidroksi-2-oksokinolin-3-il)-2,4-dioksobutanoat (1) bileşiğinin bir seri azotlu ve/veya karbonlu nükleofillerle tepkimesinden etkili bir şekilde sentezlenmiştir. Kinolin grubu içeren pirazoller, pirimidinler, pirazinler, oksazinler ve triazinler gibi bir seri heterosiklik sistemler elde edilmiştir. Yeni sentezlenmiş ürünlerin yapıları analitik ve spektral veriler temelinde doğrulanmıştır.

Anahtar Kelimeler: Kinolin-2-on, diketoester, aktif metilen, nükleofil reaksiyon, heterohalkalaşma.

Gönderilme tarihi: 12 Aralık 2015. Düzeltme tarihi: 11 Ocak 2016. Kabul tarihi: 7 Şubat 2016. 